\title{
Productive HIV-1 infection of human cervical tissue ex vivo is associated with the secretory phase of the menstrual cycle
}

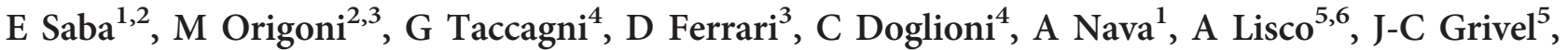 \\ L Margolis $^{5}$ and G Poli ${ }^{1,2}$
}

Cervical tissue explants (CTEs) from 22 HIV-1 seronegative women were exposed to R5 HIV-1 ex vivo. Eight CTEs were productively infected in terms of HIV-1 p24 $4_{\text {Gag }}$ release in culture supernatants, whereas 14 were not. Nonetheless, both accumulation of HIV-1 gag DNA and of $\mathrm{p} 24_{\mathrm{Gag}}^{+} \mathrm{CD} 4^{+}$T cells and macrophages occurred in both productive and, at lower levels, in nonproductive CTEs. Nonproductive CTEs differed from productive CTEs for higher secretion of C-C motif chemokine ligand 3 (CCL3) and CCL5. A post-hoc analysis revealed that all productive CTEs were established from women in their secretory phase of the menstrual cycle, whereas nonproductive CTEs were derived from women either in their secretory $(28 \%)$ or proliferative $(36 \%)$ menstrual cycle phases or with an atrophic endometrium (36\%). Thus, our results support the epidemiological observation that sexual HIV-1 transmission from males to women as well as from women to men is more efficient during their secretory phase of the menstrual cycle.

\section{INTRODUCTION}

Most of the newly HIV type-1 (HIV-1) infected women acquire infection through vaginal intercourse in which semen-associated virus is deposited in the mucosa of the lower female genital tract before infecting primary HIV-1 target cells and disseminating to the regional lymph nodes. ${ }^{1,2}$ The lack of understanding of the basic mechanisms of HIV-1 transmission and dissemination in the female lower genital tract is a limitation to developing efficient protection measures as evidenced from the recent controversial results on the efficacy of vaginal microbicides. ${ }^{3,4}$

In order to reach its primary cell targets in the female lower genital tract, i.e., $\mathrm{CD}^{+}$T lymphocytes, dendritic cells (DC), and sub-epithelial macrophages, ${ }^{5-7}$ the virus must circumvent mucosal barriers. ${ }^{8}$ The nature of these barriers is both physical (columnar or multi-stratified epithelial layer, mucus, and acidic $\mathrm{pH}$ ) and biological, including secreted factors endowed with anti-HIV activity such as C-C motif chemokine receptor 5 (CCR5)-binding chemokines, secretory leukocyte peptidase inhibitor (SLPI), and $\beta$-defensins. ${ }^{9-11}$ Notably, these mucosal barrier components as well as the state of activation of HIV-1 target cells are strongly influenced by the menstrual cycle that is under the control of sexual hormones. ${ }^{12-16}$ In this regard, different studies performed in non-human primates (NHP) have demonstrated that different phases of the menstrual cycle affect simian immunodeficiency virus (SIV) transmission and infection at the level of the cervico-vaginal mucosa. ${ }^{17,18}$ In humans, Heffron et al. ${ }^{19}$ have recently demonstrated that systemic administration of hormonal contraceptives can increase significantly the risk of HIV-1 acquisition by women as well as the likelihood of viral transmission from infected women to men.

In vitro studies using a reconstituted mucosal barrier have provided new insights in sexual transmission of HIV-1/SIV. ${ }^{20}$ Another fruitful experimental model to study the mechanisms of the early events in HIV interaction with the genital mucosa is represented by human cervical (or cervico-vaginal) tissue explants (CTEs), which retain the in vivo cyto-architecture and some tissue functions for several days in culture. ${ }^{21,22}$ By adopting this latter experimental model, we have previously reported that activated tissue-associated $\mathrm{CD}^{+}{ }^{+} \mathrm{T}$ cells are

\footnotetext{
${ }^{1}$ AIDS Immunopathogenesis Unit, Division of Immunology, Transplantation and Infectious Diseases, San Raffaele Scientific Institute, Milano, Italy. ${ }^{2}$ Università Vita-Salute San Raffaele, School of Medicine, Milano, Italy. ${ }^{3}$ Department of Obstetrics and Gynecology, San Raffaele Scientific Institute, Milano, Italy. ${ }^{4}$ Department of Pathology, San Raffaele Scientific Institute, Milano, Italy. ${ }^{5}$ Program in Physical Biology, Eunice Kennedy-Shriver National Institute of Child Health and Human Development, National Institutes of Health, Bethesda, Maryland, USA and ${ }^{6}$ Present address: University Hospitals, Case Western Reserve University, Cleveland, Ohio, USA. Correspondence: L Margolis (margolis@helix.nih.gov); G Poli (poli.guido@hsr.it)
}

Received 17 August 2012; accepted 21 December 2012; published online 6 February 2013. doi:10.1038/mi.2013.2 

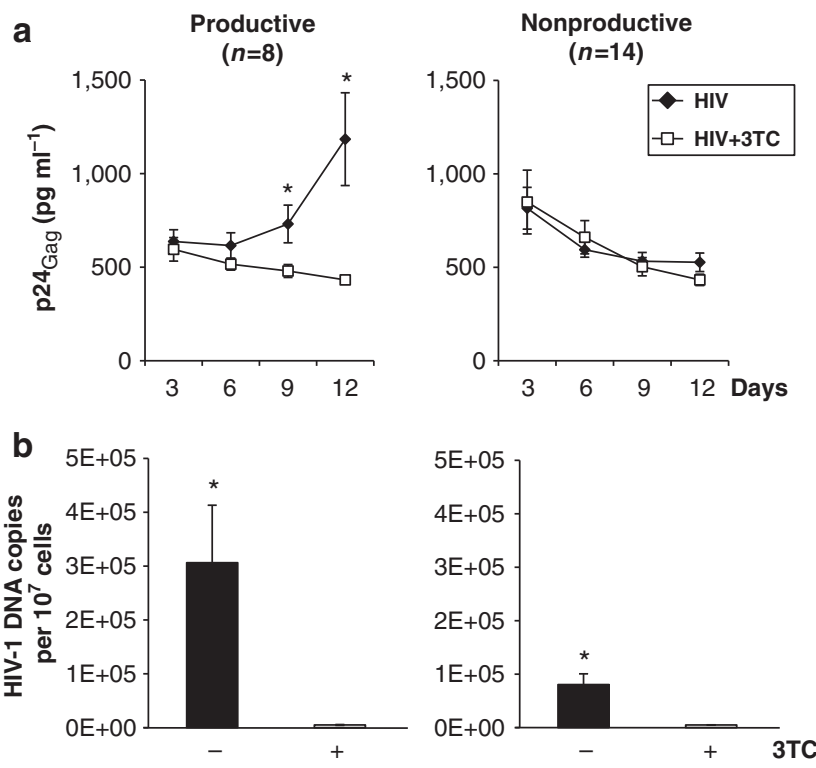

Figure 1 Productive and nonproductive R5 HIV-1 infection of cervical tissue explants (CTEs) established from seronegative women.

Donor-matched blocks of human cervical tissue were inoculated ex vivo with R5 HIV-1 $1_{\mathrm{BaL}}$ in the presence or absence of lamivudine (3TC; $5 \mu \mathrm{M}$ ). (a) Kinetics of p24 $4_{\text {Gag }}$ release in culture supernatants of productive and nonproductive CTEs (means \pm s.e.m.SEM of 8 productive and 14 nonproductive tissues; ${ }^{*} P \leqslant 0.05$, Student's $t$-test for $3 T C$ untreated vs. treated CTEs). (b) HIV-1 gag DNA levels determined in productive and nonproductive CTEs 12 days post infection in the presence or absence of 3TC (means \pm s.e.m. of 8 productive and 14 nonproductive CTEs; ${ }^{\star} P \leqslant 0.05$, Student's $t$-test between productive and nonproductive CTEs inoculated with HIV-1 in the absence of 3TC).

major targets and the likely source of CCR5-dependent (R5), but not CXCR4 (C-X-C motif chemokine receptor 4)dependent (X4), HIV $-1{ }^{22}$ Although the role of $\mathrm{CD} 4{ }^{+} \mathrm{T}$ lymphocytes in HIV-1 infection of the cervico-vaginal compartment has been thoroughly investigated, the potential contribution of resident macrophages is much less defined. ${ }^{23,24}$

Therefore, we investigated here the potential role of both $\mathrm{CD}^{+}{ }^{+} \mathrm{T}$ lymphocytes and macrophages in sustaining R5 HIV1 replication in CTEs obtained from HIV-seronegative women undergoing hysterectomy for benign gynecological conditions. We found that both cell types contribute to propagate HIV-1 infection in this model system. Furthermore, we observed that the menstrual cycle phases of the donors at the time of hysterectomy strongly affected virus production (as measured by $\mathrm{p} 24_{\text {Gag }}$ release) in CTEs. In fact, only tissues obtained from women in their secretory phase (dominated by progesterone) at the time of surgery sustained productive virus replication, whereas nonproductive infection of CTEs was observed with tissues obtained from women in either their secretory or proliferative phase of the menstrual cycle or with an atrophic endometrium.

\section{RESULTS}

R5 HIV-1 efficiently replicates in a subset of CTEs established from seronegative women

Fresh human cervical tissues (collected $\leqslant 1 \mathrm{~h}$ after surgery) were dissected into approximately $2-\mathrm{mm}^{3}$ blocks and then cultured on collagen sponge gel rafts for up to 12 days, as described. $^{21,22}$ CTEs were inoculated with R5 HIV-1 $1_{\text {BaL }}$, and virus replication was evaluated by both $\mathrm{p} 24_{\text {Gag }}$ release into the culture supernatant as well as by the accumulation of HIV DNA in tissue-associated cells. ${ }^{22}$ In order to discriminate between the viral inoculum captured nonspecifically by the tissue blocks and de novo virus replication, control donor-matched tissue blocks were incubated with R5 HIV-1 in the presence of the reverse transcriptase (RT) inhibitor lamivudine (3TC; $5 \mu \mathrm{M}$ ), as described. ${ }^{22}$

Virus replication became evident on day 9 post-HIV-1 inoculation and increased up to day 12 as evaluated by $\mathrm{p} 24_{\mathrm{Gag}}$ release in CTE culture supernatants. By contrast, there was no increase of virus release in 3TC-treated HIV-1-exposed cultures. CTEs from different donors showed a clear-cut difference in their capacity to support virus production. We defined as "productive" those CTEs in which a progressive accumulation of p24 $4_{\text {Gag }}$ into culture supernatants occurred in comparison to 3TC-treated autologous cultures. Conversely, "nonproductive" CTEs were defined as those showing no difference in $\mathrm{p} 24_{\text {Gag }}$ release in the presence or absence of 3TC. Overall, out of 22 CTEs (each established from a different donor) 8 were classified as productive, whereas the remaining 14 were defined as nonproductive (Figure 1a).

\section{Higher levels of HIV-1 DNA accumulate in productive than in nonproductive CTEs}

In order to further investigate whether CTEs established from different donors were susceptible or resistant to HIV-1 infection and replication, HIV-1 DNA was quantified by means of RT-PCR after 12 days of infection. Productive CTEs showed accumulation of HIV DNA that reached $3.06 \pm$ $1.06 \times 10^{5}$ copies of HIV- $1_{\text {gag }}$ DNA per $10^{7}$ cells over 3 TCtreated control cultures (that were essentially negative for HIV-1 DNA detection). In comparison to productive CTEs, significantly lower levels of HIV DNA $\left(0.80 \pm 0.20 \times 10^{5}\right)$ were detected in nonproductive CTEs $(P<0.05$; Figure 1b).

\section{Phenotype and susceptibility to HIV-1 infection of CTE- associated $\mathrm{CD}^{+} \mathrm{T}$ cells and resident macrophages}

In order to characterize which potential HIV-1 target cells were present in our CTEs, fresh cervical tissues from some donors were digested with collagenase IV and the cell suspensions were stained for different surface antigens. A flow cytometric analysis indicated that the percentage of $\mathrm{CD}^{+}{ }^{+} \mathrm{T}$ cells and resident macrophages (defined as lineage negative, $\mathrm{CD} 3^{\text {neg }}$, $\mathrm{CD} 19^{\text {neg }}, \mathrm{CD} 20^{\text {neg }}, \mathrm{CD} 56^{\text {neg }}, \mathrm{CD} 14^{+} \mathrm{CD} 11 \mathrm{~b}^{+}$cells) isolated from CTEs were not significantly different between productive and nonproductive tissues (data not shown). Most $\mathrm{CD}^{+}$ $\mathrm{CD} 4{ }^{+} \mathrm{T}$ cells isolated from either productive or nonproductive CTEs were CCR5 ${ }^{+}$(76.7 $\pm 3.4 \%$ vs. $81.7 \pm 12.5 \%$, respectively; $n=7$; Figure 2a) and showed an "effector memory" phenotype, as defined by the lack of expression of CCR7 and CD45RA ( $92.1 \pm 3 \%$ vs. $93.5 \pm 0.9$ for productive and nonproductive CTEs, respectively; $n=6$; Figure $2 \mathbf{b}$ ).

Different populations of resident macrophages could be distinguished based on the expression of CD4 and CCR5. 

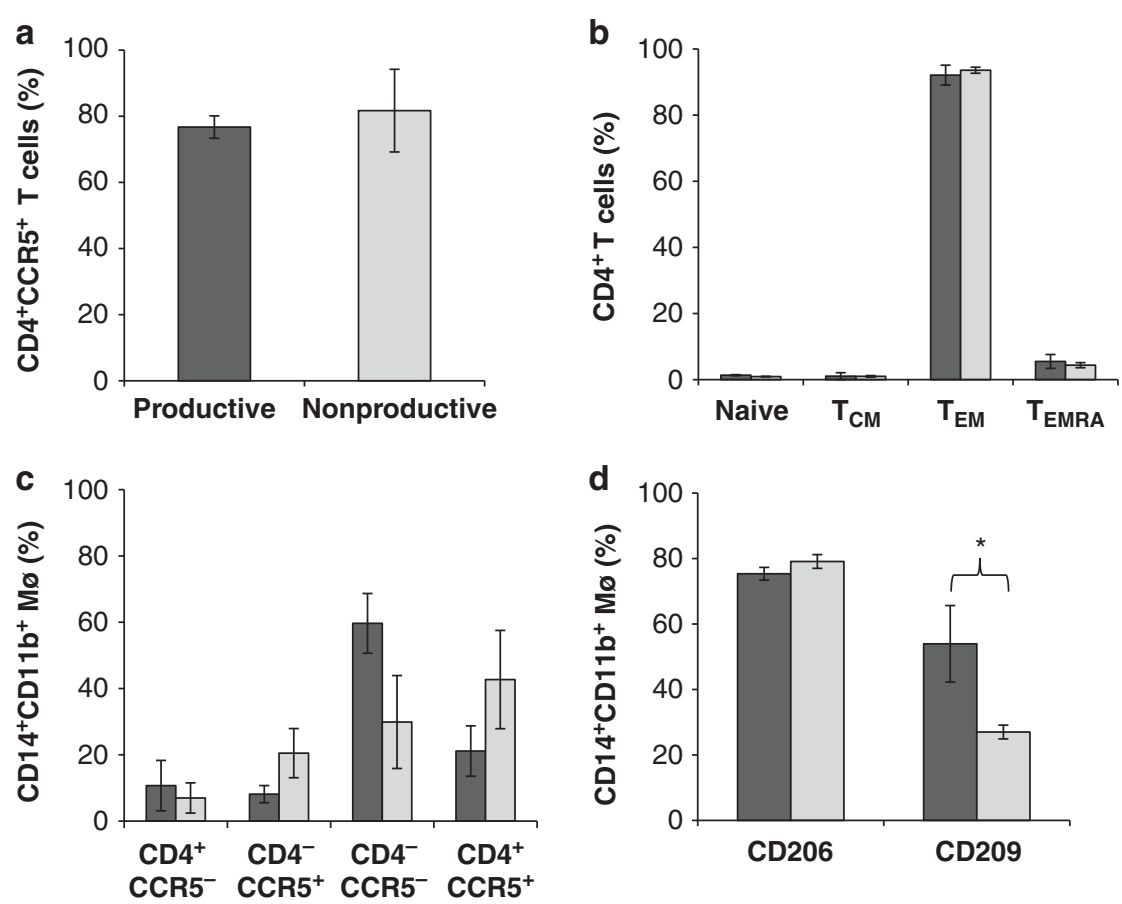

$\square$ Productive $\square$ Nonproductive

Figure 2 Phenotypic characterization of $\mathrm{CD} 4{ }^{+} \mathrm{T}$ cells and macrophages isolated from productive and nonproductive cervical tissue explants (CTEs). (a) Most $\mathrm{CD}^{+}{ }^{+} \mathrm{CD} 4^{+} \mathrm{T}$ cells present in CTEs expressed C-C motif chemokine receptor 5 (CCR5) irrespective of the productive or nonproductive outcomes of tissue infection (means \pm s.e.m., $n=7$ ). (b) $C D 3^{+} \mathrm{CD} 4^{+}$T cells were evaluated for the expression of CCR7 and CD45RA in order to define functional subsets. Most CD3 ${ }^{+} \mathrm{CD} 4^{+} \mathrm{T}$ cells present in CTEs showed an effector memory phenotype that were similarly represented in productive and nonproductive CTEs. Furthermore, no differences were observed in the fraction of naive $\left(\mathrm{T}_{\mathrm{EM}}\right.$; $\left.\mathrm{CCR} 7^{+} \mathrm{CD} 45 \mathrm{RA}{ }^{+}\right)$, central memory $\left(\mathrm{T}_{\mathrm{CM}} ; \mathrm{CCR} 7^{+}\right.$CD45RA $\left.{ }^{\text {neg }}\right)$, effector memory $\left(\mathrm{CCR} 7^{\text {neg }} \mathrm{CD} 45 \mathrm{RA}{ }^{\text {neg }}\right)$, or terminal effector memory $\left(\mathrm{T}_{\mathrm{EMRA}} ; \mathrm{CCR} 7^{\text {neg }} \mathrm{CD} 45 \mathrm{RA}{ }^{+}\right) \mathrm{CD}^{+}{ }^{+} \mathrm{CD} 4^{+} \mathrm{T}$ cells between productive and nonproductive CTEs (means \pm s.e.m., $n=6$ ). (c) Only a fraction of resident macrophages, identified $\mathrm{as} \mathrm{Lin}^{\text {neg }} \mathrm{CD} 14^{+} \mathrm{CD} 11 \mathrm{~b}{ }^{+}$, co-expressed CD4 and CCR5 irrespective of the infection outcomes of the CTEs (means \pm s.e.m., $n=8$ ). Also the remainder subsets as defined by either the expression or lack of expression of CD4 and/or of CCR5 showed a similar distribution in productive and nonproductive CTEs. (d) Resident macrophages were also positive for CD206 (means \pm s.e.m., $n=4$ ) irrespective of the productive or nonproductive outcomes of tissue infection. Conversely, productive CTEs were characterized by a higher fraction of CD209+ macrophages compared with nonproductive CTEs (means \pm s.e.m., $n=9$, ${ }^{\star} P \leqslant 0.05$, Student's $t$-test).

A minority of these cells co-expressed CD4 and CCR5 without significant differences between productive and nonproductive CTEs $(21.1 \pm 7.6 \%$ and $42.7 \pm 14.8 \%$ for productive and nonproductive CTEs, respectively; Figure 2c), as observed for T cells. Other resident macrophages expressed either CD4 alone or CCR5 alone $(10.7 \pm 7.5 \%$ vs. $6.9 \pm 4.5 \%$ and $8.1 \pm 2.5 \%$ vs. $20.5 \pm 7.4 \%$ for productive and nonproductive CTEs, respectively, Figure 2c). Finally, a macrophage subset was negative for the surface expression of both CD4 and CCR5 $(59.6 \pm 9.1 \% \quad 29.8 \pm 14.1 \%$ vs. for productive and nonproductive CTEs, respectively; Figure 2c), thus resembling a phenotype described for intestinal macrophages. ${ }^{6}$

Resident macrophages were also evaluated for the expression of mannose receptor (CD206) and of dendritic cell-specific intercellular adhesion molecule-3-grabbing non-integrin (DCSIGN, CD209). No differences were observed in the fractions of $\mathrm{CD} 206^{+}$macrophages between productive and nonproductive CTEs (75.3 \pm 1.9 vs. $79.1 \pm 2,1$ respectively; $n=4$; Figure $2 d$ ). By contrast, a higher percentage of $\mathrm{CD} 209^{+}$macrophages was observed in productive vs. nonproductive CTEs $(53.9 \pm 11.6 \%$ vs. $27 \pm 2.1 \%$ respectively; $n=9, P<0.05$; Figure 2d).
Infection of HIV-1 target cells present in the CTEs was confirmed by intracellular p24 $4_{\mathrm{Gag}}$ staining of cells isolated from tissue blocks obtained from the 22 different donors 6 days after infection (Figure 3a). A trend of higher frequency of p24 $4_{\text {Gag }}^{+}$ $\mathrm{CD} 8^{\text {neg }} \mathrm{T}$ cells (that were gated in order to include $\mathrm{CD} 4^{+} \mathrm{T}$ lymphocytes that downregulated $\mathrm{CD} 4$ as a consequence of HIV-1 infection ${ }^{22}$ ) and macrophages was observed in productive vs. nonproductive CTEs, although these differences were not statistically significant $(14.2 \pm 3.2 \%$ vs. $8.1 \pm 2.3 \%$ for productively and nonproductively infected CTEs, respectively, for $\mathrm{T}$ cells, and $1.7 \pm 0.7 \%$ vs. $0.6 \pm 0.3 \%$, respectively, for macrophages; Figure 3b).

\section{Comparable levels of anti-viral peptides and pro- inflammatory cytokines are secreted by both productively and nonproductively infected and uninfected CTEs}

Both $\beta$-defensins and SLPI have been associated with anti-HIV activities, particularly at the mucosal level. ${ }^{9,10}$ Therefore, we investigated whether the secretion of these innate antiviral molecules could explain the different outcomes of CTE infection. However, no differences in the release of these 

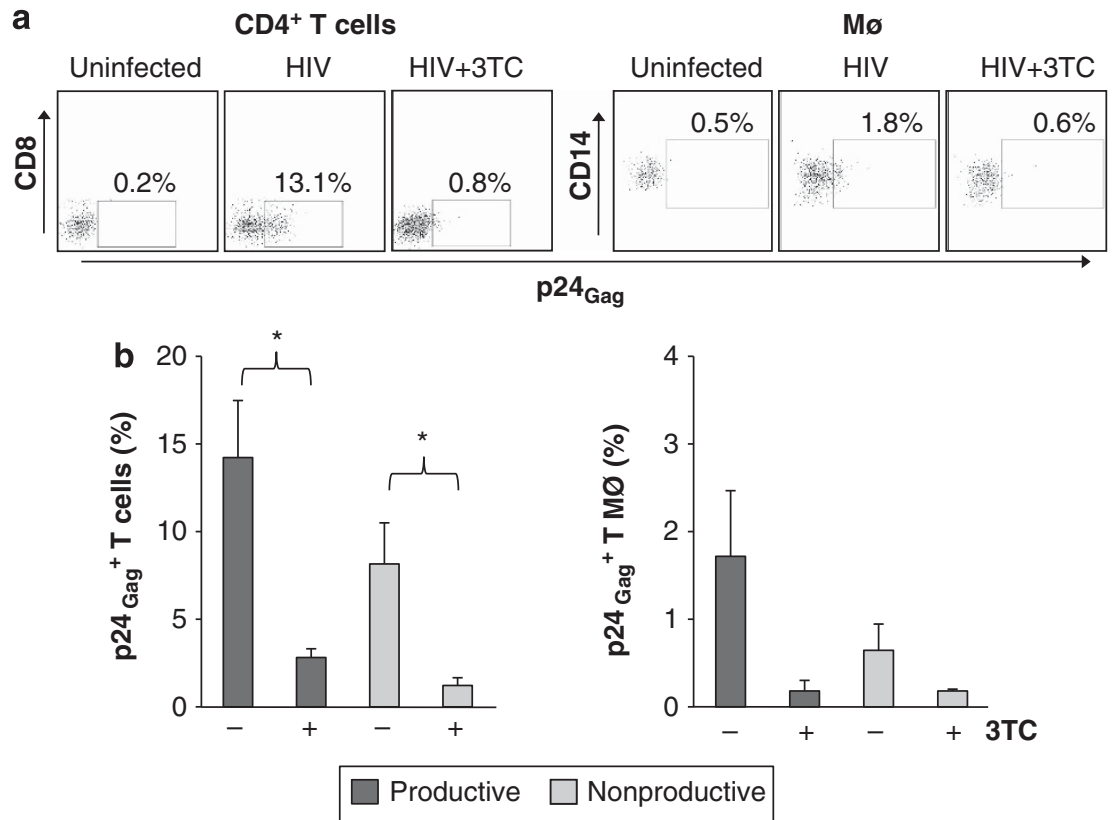

Figure 3 Both cervical tissue explant (CTE)-associated T cells and macrophages express HIV-1 p24Gag. HIV-1 p24 ${ }_{\text {Gag }}^{+}$CD $3^{+}$CD $8^{\text {neg }}$ T cells and $\mathrm{Lin}^{\text {neg }} \mathrm{CD} 14^{+} \mathrm{CD}_{11 \mathrm{~b}}{ }^{+}$macrophages were detected in donor-matched CTEs infected with R5 HIV-1 $1_{\mathrm{BaL}}$ in the presence or absence of lamivudine (3TC; $5 \mu \mathrm{M}$ ) 6 days post infection. (a) Bivariate density plots of a single experiment with cells isolated from the CTEs of one donor representative of eight with a productive infection outcome. (b) T cells and macrophages isolated from both the productive and nonproductive CTEs were positive for HIV-1 p24 Gag expression at day 6 post infection; presented are the mean \pm s.e.m. data from tissues of 8 productive and 14 nonproductive CTEs. ${ }^{*} P \leqslant 0.05$, Student's $t$-test.

factors were observed between productive and nonproductive CTEs, as measured after 3 days (Figure 4, upper panel) or 12 days (data not shown) of culture both in the absence or presence of HIV-1.

As an inflammatory environment also could be involved in the differential permissiveness of CTEs to HIV-1 infection and spreading, we determined the levels of secretion of proinflammatory cytokines tumor necrosis factor- $\alpha$ (TNF- $\alpha$ ), interferon- $\gamma$ (IFN- $\gamma$ ) and C-C motif chemokine ligand 2 (CCL2; a chemokine previously reported to be associated with increased virus replication ${ }^{25,26}$ ), as well as of the anti-inflammatory cytokines interleukin-4 (IL-4) and IL-10, after 3 and 12 days from virus inoculation. Low levels of TNF- $\alpha$ and IFN- $\gamma$ were released by both productive and nonproductive CTEs irrespective of whether they were exposed or not to HIV-1 (Figure 4, middle panel), whereas the concentrations of both IL4 and IL- 10 by all CTEs were below the detection threshold of the enzyme-linked immunosorbent assay (ELISA) at both time points (data not shown). CCL2 was secreted at higher levels than cytokines, although no differences were observed between infected and uninfected CTEs or between productive vs. nonproductive CTEs (Figure 4, middle panel). Also, no differences were observed for all these analytes in the different culture conditions after 12 days of virus exposure (data not shown).

\section{Nonproductive CTEs show increased CCL3 and CCL5 secretion in comparison to productive CTEs}

Next, we evaluated whether the differential permissiveness of CTEs was associated with different levels of secreted CCR5 ligands (namely, CCL3, CCL4, and CCL5) into culture supernatants 3 and 12 days after virus inoculation or in donor-matched control tissues not exposed to HIV-1. In contrast to what was observed for antiviral peptides and cytokines, nonproductive CTEs were characterized by a higher release of CCL3 reaching statistical significance in uninfected conditions $\left(103.7 \pm 13.9\right.$ vs. $175.5 \pm 32.7 \mathrm{pg} \mathrm{ml}^{-1}$ for productive and nonproductive CTEs, respectively; $P<0.05$; Figure 4, lower panel). HIV-1 inoculation was associated with significantly higher levels of CCL5 in nonproductive vs. productive CTEs $(11.5 \pm 1.1$ vs. $26.7 \pm 3.4$ and $13.2 \pm 1.7$ vs. $30.6 \pm 4.1 \mathrm{pg} \mathrm{ml}^{-1}$ for productive and nonproductive CTEs infected in the absence or presence of 3TC, respectively, $P<0.05$; Figure 4, lower panel) 3 days after virus exposure. Moreover, we compared the levels of secreted CCL5 released from four nonproductive tissues with those of the eight productive ones after HIV-1 infection, all obtained from the donors in the secretory phase. On average, the levels of CCL5 secreted by nonproductive tissue upon HIV-1 infection were twice as high than those of productive tissue, although this difference did not reach statistical significance likely due to the small numbers of donors in these two groups (data not shown). These differences were not observed after 12 days of CTE cultures both in the presence or absence of HIV-1 (data not shown).

Only CTEs from women in their secretory phase of the menstrual cycle became productively infected with R5 HIV-1 In order to better understand whether the productive or nonproductive outcomes of CTE infection correlated with a 

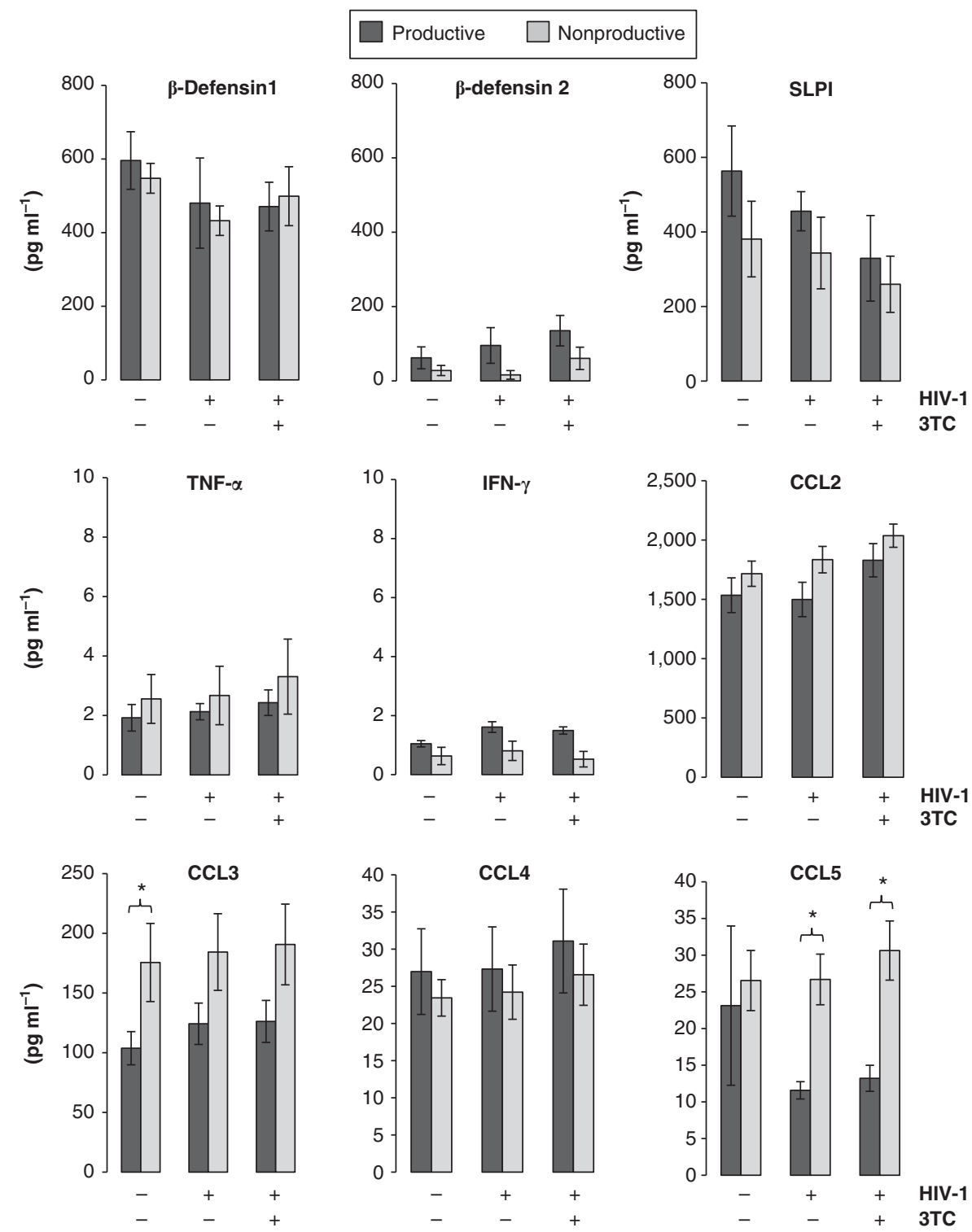

Figure 4 Selective upregulation of C-C motif chemokine ligand 3 (CCL3) and CCL5 release in the supernatant of nonproductive vs. productive cervical tissue explants (CTEs). CTEs were exposed to R5 HIV-1 $1_{\mathrm{BaL}}$ in the presence or absence of lamivudine (3TC; $\left.5 \mu \mathrm{m}\right)$ and then cultured for 3 days; supernatants were collected and analyzed for the presence of the indicated analytes. The release of anti-viral peptides ( $\beta$-defensins and secretory leukocyte peptidase inhibitor (SLPI); upper panel), pro-inflammatory cytokines (tumor necrosis factor (TNF)- $\alpha$, interferon (IFN)- $\gamma$ ), or of CCL2 (middle panel) was not different in culture supernatants of productive and nonproductive CTEs. Supernatants were also analyzed for the presence of CCR5 ligands (CCL3, CCL4, CCL5; lower panel). CCL3 production was significantly higher in uninfected CTEs showing a nonproductive profile upon HIV-1 exposure, whereas CCL5 was significantly released more by HIV-1-exposed nonproductive vs. productive CTEs both in the presence or absence of 3 TC. Presented are means \pm s.e.m. from 8 productive and 14 nonproductive CTEs. ${ }^{*} P \leqslant 0.05$, Student's $t$-test.

different tissue architecture, we performed a post-hoc histological analysis of the cervical tissues after surgery. In particular, the cervical tissues were fixed with $10 \%$ formaldehyde, stained with hematoxylin/eosin, and were analyzed for the presence of koilocytosis (an epithelial cell transformation that may occur as a result of infection by the human papillomavirus), connective tissue sclerosis, glandular ectasia, and metaplasia. These cervical pathological lesions were, however, similarly distributed between productive and nonproductive CTEs, except for connective tissue sclerosis that was detected exclusively in $21 \%$ of nonproductive donors (Figure 5a).
Quite remarkably, the different outcomes of HIV-1 infection (i.e., productive vs. nonproductive infection in terms of $\mathrm{p} 24_{\mathrm{Gag}}$ release in the culture supernatants) were strongly correlated with the different phases of the menstrual cycle of the donors at the time of surgical intervention. In fact, a post-hoc analysis revealed that all productive CTEs were established from women in their secretory phase at the time of surgery (Figure 5b). Conversely, as shown in Figure 5b,c, nonproductive CTEs were derived from women either in their proliferative $(36 \%)$ or secretory $(28 \%)$ phases of the menstrual cycle or histologically characterized by an atrophic 


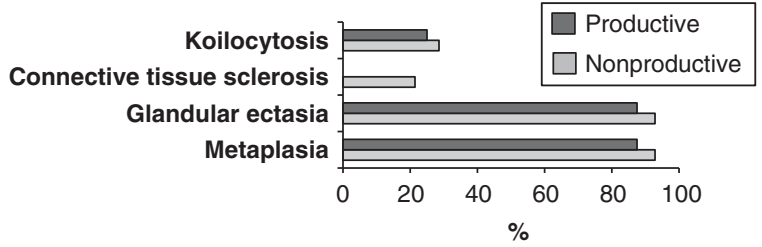

b
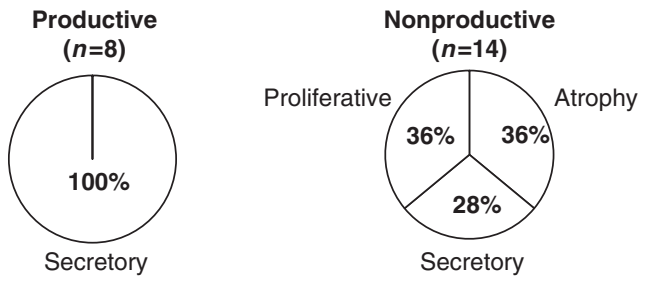

C

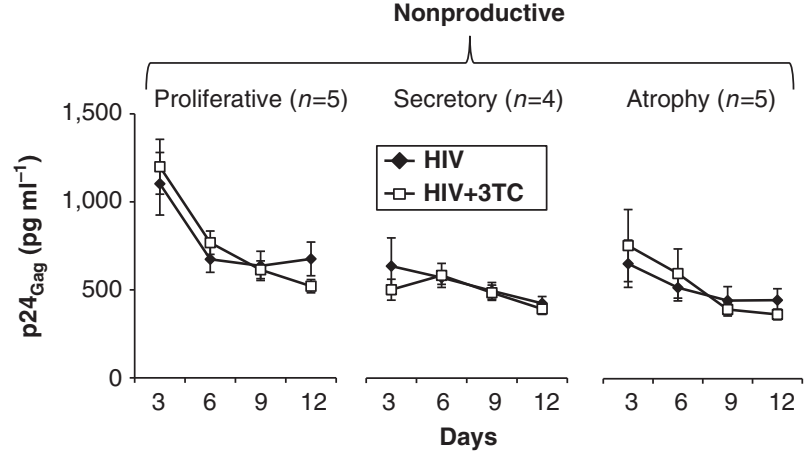

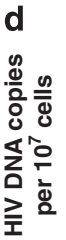
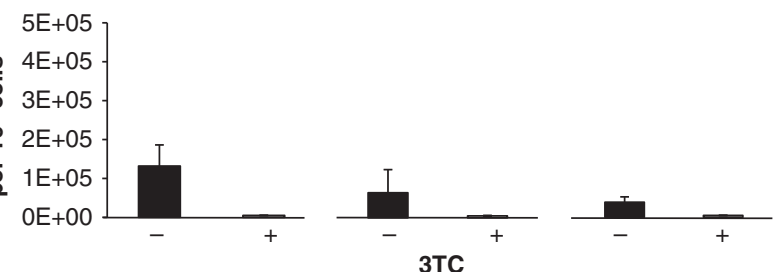

Figure 5 Association between the outcome of R5 HIV-1 exposure of cervical tissue explants (CTEs) and the donor's menstrual cycle phase or the presence of endometrial atrophy at the time of hysterectomy. (a) Histological analysis of freshly isolated cervical tissue was performed in order to analyze the presence of lesions, which included koilocytosis, connective tissue sclerosis, glandular ectasia, and metaplasia, associated with leiomyomas. The percentages of affected donors were similar, irrespective of the productive or nonproductive outcomes of CTE infection, except for connective tissue sclerosis that was present in $21 \%$ of nonproductive CTEs. (b) Only tissues from women in their secretory phase were productively infected by R5 HIV-1 $1_{\text {BaL }}$, as shown in Figure 1a. (c) Undistinguishable p24 $4_{\mathrm{Gag}}$ release between untreated and lamivudine (3TC)-treated CTEs by nonproductive CTEs. CTEs from donors in proliferative phase showed a higher initial level of $\mathrm{p} 24_{\text {Gag }}$ than those from donors in secretory phase or with an atrophic endometrium, likely as a reflection of the different mucus composition. (d) HIV-1 DNA accumulation occurred in nonproductive CTEs; no significant differences were noted in nonproductive CTEs in terms of HIV-1 DNA levels according to the menstrual cycle phase or the presence of endometrial atrophy.

endometrium (36\%). Nonproductive CTEs, however, showed low but detectable levels of HIV-1 DNA accumulation above the 3 TC controls $\left(1.31 \pm 0.54,0.64 \pm 0.01,0.39 \pm 0.01 \times 10^{5}\right.$ copies of HIV $-1_{\text {gag }}$ DNA for the proliferative or secretory phases or endometrial atrophy, respectively; Figure 5d). No differences were observed in the age of donors from whom productive or nonproductive CTEs were established, except for those with an atrophic endometrium who were significantly older than the others, as detailed in Table 1.

\section{DISCUSSION}

In order to infect its target cells in the course of male-to-female HIV-1 transmission, the virus must cross the mucosal barrier, consisting of an epithelial layer covered by mucus. ${ }^{8}$ The exact mechanisms of this process and the nature of HIV-1 cell targets, especially the involvement of macrophages resident in the submucosa, ${ }^{7}$ remain largely speculative. Moreover, all these barrier elements are strongly influenced by the menstrual cycle that is under the control of sexual hormones. ${ }^{12-14}$ Accordingly, susceptibility of women to viral infections depends on their hormonal status as experimentally demonstrated by SIV infection of NHP. ${ }^{17,18}$ Of note, a significant increase of the risk of HIV-1 acquisition associated with a higher likelihood of female-to-male transmission has been reported in women taking or women using hormonal contraceptives systemically. ${ }^{19}$ In order to decipher the mechanisms of HIV-1 transmission leading to the infection of target cells in the female lower genital tract, it is crucial to investigate these phenomena in an adequate laboratorycontrolled system ex vivo, such as the R5 HIV-1 infection of CTEs used in the present study. In fact, the cyto-architecture and at least some of the tissue cell functions are maintained in CTEs for several days in culture; furthermore, CTEs are permissive for productive R5, although not X4, HIV-1 infection without requiring exogenous stimulation by mitogens or cytokines. ${ }^{21,22}$

In the present study, we have investigated the susceptibility to productive R5 HIV-1 infection of CTEs established ex vivo from 22 HIV-1 seronegative women undergoing hysterectomy for the presence of non-malignant tumors. Here, we focused exclusively on the exposure of CTEs to R5, and not to X4 HIV-1, as in vivo R5 HIV-1 dominates the earliest stages of HIV infection following sexual transmission. Furthermore, ex vivo R5 HIV-1 but not X4 HIV-1 productively infects CTEs, as we have reported earlier. ${ }^{22}$ In the present study, the primary criterion for defining a productive infection was the release of $\mathrm{p} 24_{\mathrm{Gag}}$ above the baseline release in donor-matched cultures inoculated with HIV-1 in the presence of the RT inhibitor 3TC. HIV-1-exposed cultures in the presence of 3TC, indeed, gradually released p24 $4_{\text {Gag }}$ in the supernatant that we considered as HIV-1 background. According to this criterion, 8 out of 22 CTEs (36\%) showed clear-cut evidence of virus replication, whereas in the remainder cultures the levels of total p24 $4_{\text {Gag }}$ released in the presence or absence of 3TC were superimposable. Thus, the outcome of HIV-1 inoculation of CTEs fell into two clear-cut categories, i.e., "productive" and "nonproductive" infections.

Both $\mathrm{CD}^{+} \mathrm{T}$ cells and resident macrophages supported HIV-1 replication, although macrophages (only a minority of which co-expressed CD4 and CCR5) to a lesser extent than T lymphocytes. At present, however, we cannot exclude the possibility that these resident macrophages merely took up virions earlier produced by $\mathrm{CD} 4^{+} \mathrm{T}$ lymphocytes, rather than being productively infected. The different outcomes of infection (i.e., productive vs. nonproductive) did not correlate with the number of HIV-1 target cells; in fact, the relative numbers of 
Table 1 Endometrial histology and HIV-1 infection outcomesNA, not applicable.

\begin{tabular}{|c|c|c|c|c|}
\hline HIV infection ${ }^{a}$ & Number of donors & \multicolumn{3}{|c|}{ Endometrial histology } \\
\hline Productive & 8 & 0 & 8 & 0 \\
\hline Nonproductive & 14 & 5 & 4 & 5 \\
\hline Age (range) (years) & $55 \pm 3(40-76)$ & $50 \pm 2(40-56)$ & $50 \pm 6(47-51)$ & $65 \pm 9(55-76)$ \\
\hline
\end{tabular}

CCR5-expressing $\mathrm{CD}^{+} \mathrm{T}$ cells and macrophages were comparable in both productive and nonproductive CTEs. Furthermore, the functional distributions of $\mathrm{CD} 4{ }^{+} \mathrm{T}$ cells were similar in productive and nonproductive CTEs, with CD4 ${ }^{+} \mathrm{T}$ cells displaying an "effector memory" phenotype representing the dominant subset. In general, our findings are in agreement with the report of Shen et al. ${ }^{27}$ who isolated DC, CD4 ${ }^{+}$T cells, and macrophages from ectocervical tissue, infected them in vitro with an R5 HIV-1 strain, and found that all these cells were productively infected although with different kinetics.

Concerning resident macrophages, although the percentage of those expressing CD206 (mannose receptor) was comparable in both productive and nonproductive CTEs, there was a significantly higher percentage of cells expressing CD209 (DCSIGN) on their surface in productive CTEs than in nonproductive ones. In this regard, DC-SIGN has been described as an HIV-1 "capture" receptor capable of promoting infection of $\mathrm{CD}^{+}$T cells in trans ${ }^{28,29}$ as well as of influencing cis infection of DC via triggering of a signal transduction pathway involving Raf- $1 .{ }^{30}$ Although the role of DC-SIGN in the biology of DC has been thoroughly investigated, the potential consequence of its expression by resident macrophages in the context of sexual HIV-1 transmission is much less understood. The observation of a more frequent expression of DC-SIGN by resident cervical macrophages of those CTEs showing a productive profile upon infection suggests its potential contribution to HIV propagation in the tissue. In this regard, it is of interest that the potential efficacy of a candidate microbicide targeting DC-SIGN and preventing HIV-1 infection in cellular and human cervical explant models has been recently reported. ${ }^{31}$

Searching for biological correlates of productive and nonproductive CTE infections, however, we did not observe significant differences in terms of secreted levels of either innate anti-viral peptides ( $\beta$-defensins, SLPI), pro-inflammatory (TNF- $\alpha$, IFN- $\gamma$, CCL2), or anti-inflammatory (IL-4, IL-10) cytokines. By contrast, some CCR5-binding chemokines were secreted at higher levels by nonproductive vs. productive CTEs. In the case of CCL3, this difference was statistically significant in uninfected tissues, whereas for CCL5 the difference between productive and nonproductive CTEs became evident after infection. Although this observation is only correlative, in consideration of the well-established role of CCR5-binding chemokines in inhibiting HIV-1 infection (in all the main routes of transmission, sexual, mother-to-child, blood-related ${ }^{32}$ ), we speculate that the higher levels of CCL3 and CCL5 released by nonproductive CTEs might have contributed to prevent or curtail R5 HIV-1 infection in these tissues, including nonproductive CTEs established from women in their secretory phase (in the case of CCL5), at least during the initial days following HIV-1 exposure, as no differences were observed after 12 days of culture. This interpretation fits with the observation of significantly higher levels of CCL5 in the genital secretion of female commercial sex workers naturally resistant to HIV-1 infection compared with those of uninfected commercial sex workers; ${ }^{33}$ however, no information on whether the levels of the chemokine varied according to the menstrual cycle was reported in this study. ${ }^{33}$ A broader upregulation of chemokine release in vitro by either productive infection or by exposure to HIV-1 proteins (including Tat, Nef, and gp120 Env) has been previously described in cultures of either activated PBMC (peripheral blood mononuclear cells), monocyte-derived macrophages, or glial cell lines. ${ }^{34-37}$ Thus, our findings sustain the potential relevance of strategies aimed at blocking R $5 \mathrm{HIV}-1$ access to the CCR 5 co-receptor in order to hamper early local replication in the female genital tract, an event also influenced by hormonally mediated modulation of the CCL5/CCR5 axis. ${ }^{38}$ In this regard, higher levels of CCR 5 expression by T cells in the uterine cervix and blood obtained from post-menopausal women have been recently reported. ${ }^{39}$

A post-hoc analysis of the ex vivo outcomes of HIV-1 exposure of CTEs (productive vs. nonproductive) did not reveal a correlation with histological alterations of the mucosa and submucosa. In particular, we evaluated the tissues immediately after surgery for the presence of koilocytosis, connective tissue sclerosis, glandular ectasia, and metaplasia. No differential distribution of these cervical lesions were observed with the exception of connective tissue sclerosis, a condition associated with an atrophic endometrium, typical of a menopausal state, that was observed exclusively in $21 \%$ of nonproductive CTEs. By contrast, a strong correlation was observed between productive HIV-1 infection of CTEs and the menstrual cycle phase of the donor at the time of surgery. Remarkably, all CTEs that were productively infected were obtained from women in their secretory phase (dominated by progesterone), whereas nonproductive tissues were obtained from women in different phases of their menstrual cycle, i.e., secretory (28\%) or proliferative (36\%) phases (the latter dominated by estrogens) or characterized by an atrophic endometrium (36\%), a condition associated with the virtual absence of sexual hormones as typically observed in menopause. The strong 
association between the secretory phase of the menstrual cycle and the productive infection outcome of CTEs was supported by the observation of higher levels of HIV-1 DNA accumulation in productive vs. nonproductive CTEs.

The menstrual cycle and its related sexual hormones are known to be important factors in sexual HIV-1 transmission as highlighted by studies of SIV transmission in Rhesus macaques that are frequently treated with progesterone derivatives (such as Depoprovera) in order to increase the efficiency of intravaginal infection. ${ }^{40}$ Although these studies indicate that progesterone did not influence the number of SIV target cells in the NHP female genital tract, ${ }^{41}$ the hormonal treatment induced an increased tightness of the vaginal epithelium, ${ }^{12}$ disruption of the integrity of epithelial tight junctions, ${ }^{14}$ and decreased the production of antimicrobial molecules, including SLPI $^{42}$ and $\beta$-defensins. ${ }^{43,44}$ Concerning HIV- 1 infection, Heffron et al. ${ }^{19}$ reported that the use of injectable formulation of hormonal contraceptives based on progestinic hormones in African women increased both their frequency of HIV-1 acquisition and the likelihood of transmitting the infection to their male partners. In this regard, our results of ex vivo infection of CTEs are in full agreement with both the epidemiological and the clinical experimental evidence that the secretory phase of the menstrual cycle (controlled by progesterone) is associated with the release of $\mathrm{p} 24_{\mathrm{Gag}}$, likely reflecting virion production, and, consequently, with a higher likelihood of viral transmission by sexual intercourse.

In summary, our results demonstrate an association between the capacity of the cervical tissue to support productive HIV-1 infection, at least ex vivo, and the menstrual cycle phase of the donor, in particular with the progesterone-dependent secretory phase. Therefore, the menstrual cycle of women and/or their consumption of hormonal-based contraceptives should be taken into consideration when new preventative strategies against HIV transmission are developed and tested for their efficacy. Our study also emphasizes that CTEs can represent an adequate experimental system to study the mechanisms underlying the different susceptibility to HIV-1 infection and capacity to support its replication in different stages of the menstrual cycle. Finally, CTEs could be adopted as a low-cost platform for testing novel antivirals and microbicides before moving towards more expensive NHP models of HIV-1 transmission and clinical trials in women.

\section{METHODS}

Clinical diagnosis, histological analysis, and determination of the menstrual cycle of the donors. Cervical tissues were obtained from 22 HIV-negative women ( $52.5 \pm 8.9$ years old, range: $40-76)$ undergoing hysterectomy for non-malignant indications (i.e., multiple leiomyomas, adnexal cysts, or abnormal bleeding) in the Department of Obstetrics and Gynecology of the San Raffaele Scientific Institute, Milano, Italy. The histological analysis confirmed that all women were affected by multiple leiomyomas of the uterus. In addition, cervical tissues were evaluated for the presence of koilocytosis, connective tissue sclerosis, glandular ectasia, and metaplasia. According to surgical protocols, women were asked to stop the consumption of any pharmacological agent and, in particular, of oral contraceptives at least
1 month before surgery. A short-term broad-spectrum antibiotic prophylaxis was administrated the day of the procedure.

The uterine/menstrual cycle stage of the donors was determined on the basis of medical records and upon endometrial dating (i.e., morphological evaluation of endometrium sections stained with hematoxylin/eosin). This analysis indicated that, randomly, hysterectomy was performed in women either in their secretory $(n=12)$ or proliferative $(n=5)$ phases of the menstrual cycle; in addition, five cervical tissues were obtained from women characterized by an atrophic endometrium (Table 1).

CTE culture and ex vivo HIV-1 infection. The cervix was separated from the uterus within $60 \mathrm{~min}$ from the surgical removal and placed into cold RPMI-1640 (Lonza, Basel, Switzerland). Briefly, the mucosal epithelium and the underlying stroma of both ecto- and endo-cervix were separated from the muscular tissue and dissected into approximately $2-\mathrm{mm}^{3}$ blocks.

Fresh CTEs from 22 donors were placed in culture and infected with CCR5-dependent (R5) HIV-1 $1_{\mathrm{BaL}}$, according to a published protocol; ${ }^{22,45}$ in addition, some tissue blocks from the same clinical samples were processed for phenotyping of $\mathrm{T}$ cells and resident macrophages. Sixteen tissue blocks per condition for each donor were incubated with $500 \mu \mathrm{l}$ of undiluted R $5 \mathrm{HIV}-1_{\mathrm{BaL}}$, washed, and placed on the top of collagen sponges at the liquid-air interface.

Viral stocks were prepared using the supernatants of PBMC stimulated with phytohemagglutinin ${ }^{46}$ and IL-2 (400 $\mathrm{U} \mathrm{ml}^{-1}$; R\&D Systems, Minneapolis, MN) and infected with HIV-1 $1_{\mathrm{BaL}}$ collected at the peak of virus replication, typically occurring between the first and second week of culture, as determined by liquid phase RT activity. ${ }^{25}$ The infectious titer of the viral stock $\left(6.8 \times 10^{7} \mathrm{IU} \mathrm{ml}^{-1}\right)$ was determined on phytohemagglutinin-stimulated PBMC by applying the Reed and Muench formula. ${ }^{46}$

CTE supernatant was replaced with fresh complete medium (RPMI, $15 \%$ FBS (fetal bovine serum), 1\% L-glutamine, 1\% penicillin/ streptomycin, 1\% MEM (Minimum Essential Medium)-non-essential amino acids, $1 \%$ MEM-sodium pyruvate, $1 \%$ fungizone, $0,1 \% 1 \times$ gentamicin) every 3 days for up to 12 days of culture, after which time point the CTEs no longer maintain their morphology and viability, as reported. $^{22,47}$

De novo productive infection of CTEs was defined as the difference in $\mathrm{p} 24_{\mathrm{Gag}}$ release into the culture medium in the absence and in the presence of the RT inhibitor lamivudine (3TC; $5 \mu \mathrm{M}$ ), as reported. ${ }^{22}$

Real-time PCR for HIV-1 DNA gag quantification. DNA was extracted from tissue blocks after 12 days of incubation with HIV-1 using a Nucleospin tissue kit from MN (Duren, Germany) following the provided protocol. Real-time-PCR was performed to quantify complete viral DNA transcripts using the following primer sets that recognize the HIV-1 $1_{\text {gag }}$ gene: ${ }^{48}$ forward primer, $5^{\prime}$-ACATCAAGC AGCCATGCAAAT- $3^{\prime}$; reverse primer, $5^{\prime}$-ATCTGGCCTGGTGC AATAGG- $3^{\prime}$; and probe, $5^{\prime}$-(FAM) CATCAATGAGGAAGCTGC AGGAATGGGATAGA (TAMRA)-3'. The amount of cellular DNA was assessed by use of glyceraldehyde-3-phosphate dehydrogenase $(G A P D H)$ as a reference gene using the following primes and probe: forward primer, 5'-ACCACAGTCCATGCATCACT-3'; reverse primer, 5'-GGCCATCACGCCACAGITT- $3^{\prime}$; and probe, $5^{\prime}$-(FAM) CCCAGAAGACTGTGGATGGCCCC (TAMRA)- $3^{\prime} .{ }^{49}$ Samples were run on a Prism 7500 sequence detection system (Applied Biosystems, Foster City, CA) using a Taqman universal master mix (Life Technology, Grand Island, NY).

Immunofluorescent cytometric bead assay for HIV-1 p24 Gag quantification. Productive HIV-1 infection was assessed by measuring HIV-1 antigen $\mathrm{p} 24_{\mathrm{Gag}}$ concentration in tissue culture medium using an immunofluorescent cytometric bead assay with minor modifications from a previously described protocol. ${ }^{50}$ Briefly, carboxyl polystyrene particles (Spherotech, Lake Forest, IL) were coupled with p $24_{\text {Gag }}$ capture monoclonal antibody (mAb; Immuno Diagnostics, 
Woburn, MA) covalently using sulfo-NHS and EDC (Thermo Scientific, Rockford, IL) according to the Luminex standard protocol. Coupled microspheres $\left(4 \times 10^{3}\right)$ were consequently mixed with $50 \mu \mathrm{l}$ of standard (Perkin Elmer p24-alliance standard, Perkin Elmer, Waltham, MA) or lysed culture medium and incubated overnight at $4{ }^{\circ} \mathrm{C}$. After washing (phosphate-buffered saline plus $0.02 \%$ Tween 20 , $0.1 \%$ bovine serum albumin), an anti-p24 $4_{\mathrm{Gag}}$-phycoerythrin (PE)conjugated detection $\mathrm{mAb}$ was added (Beckman Coulter, Brea, CA) for $2 \mathrm{~h}$ at room temperature. Samples were washed and run on a LSRFortessa flow cytometer (BD Biosciences, San Jose, CA) using the DIVA 6.1.2 software (BD Biosciences); the events were analyzed with the FlowJo version 8.8.7 (Tree Star, Ashland, OR) and with the Prism 5.0 (GraphPad Software, San Diego, CA), a program using a fiveparameter fitting algorithm.

Immunophenotyping of HIV-1 target cells present in CTEs. Both T cells and macrophages were isolated after tissue digestion and staining by $\mathrm{mAb}$ directed against cell surface markers followed by cytofluorometric analysis. In particular, fresh CTEs were digested with Collagenase IV ( $5 \mathrm{mg} \mathrm{ml}^{-1}$; Life Technologies, Carlsbad, CA) in RPMI $5 \%$ FBS for $90 \mathrm{~min}$. In order to characterize tissue $\mathrm{T}$ lymphocytes, single-cell suspensions were washed in staining buffer (phosphatebuffered saline plus 2\% FBS) and stained with anti- CD3 (PE-labeled), anti-CD4 (APC), anti-CD8 (Pacific Blue), anti-CCR7 (PE-Cy7), antiCD45RA (PE-TexasRed), and/or anti-CCR5 (PE-Cy7) mAbs. In order to identify macrophages, cell suspensions obtained by tissue disruption were washed in DMEM (Dulbecco's modified Eagle's medium), 10\% FBS, 5\% human serum and then stained with FITC (fluorescein isothiocyanate)-labeled anti-CD3, anti-CD56, anti-CD19, anti-CD20 $\mathrm{mAb}$ in a single "dump" channel and with anti-CD14 (APC-Cy7), anti-CD11b (Pacific Blue), anti-CD4 (APC), and anti-CCR5 (PE-Cy7) mAbs. Macrophages were identified as lineage negative $\left(\mathrm{CD} 3^{\text {neg }}\right.$, $\left.\mathrm{CD} 19^{\text {neg }}, \mathrm{CD} 20^{\text {neg }}, \mathrm{CD} 56^{\text {neg }}\right), \mathrm{CD}_{1}{ }^{+} \mathrm{CD}_{11 b^{+}}$cells. In some experiments, macrophages were also stained with anti-CD206 (PE) and anti-CD209 (PerCP-Cy5.5). All the mAb were purchased from BD Biosciences except anti-CD8 (Pacific Blue) $\mathrm{mAb}$, which was purchased from Life Technologies.

Cell suspensions obtained by tissue digestion of 22 CTEs inoculated with HIV-1 were also collected and stained 6 days after infection with the anti-HIV-1 p24 $4_{\text {Gag }}$ KC57 mAb conjugated with FITC (Beckman Coulter, Fullerton, CA). The events were acquired by an LSR-Fortessa flow cytometer (BD Biosciences) equipped with 355-, 407-, 488-, 532-, and 638-nm laser lines using the DIVA 6.1.2 software and were then analyzed by FlowJo version 8.8.7. Dead cells were identified and excluded from the analysis using the LIVE/DEAD fixable Blue Dead Cell Stain kit (Life Technologies).

Multiplex chemokine and cytokine analysis. Supernatants from CTEs were collected 3 and 12 days after infection in the presence or absence of 3TC and analyzed with a Fluorokine MAP Multiplex Cytokine Panel (R\&D Systems). Samples were run on Bioplex 200 machine (Bio-Rad Laboratories, Hercules, CA) and analyzed by the Bioplex Manager 6 program. The CTE supernatants were tested for the presence of CCL2 (monocyte chemotactic protein-1), CCL3, CCL4 (macrophage inflammatory protein- $1 \alpha$ and $\beta$, respectively), CCL5 (regulated upon activation normal $\mathrm{T}$ cell expressed and secreted), IL-4, IL-10, TNF- $\alpha$, and IFN- $\gamma$.

$\boldsymbol{\beta}$-Defensins 1 and 2 and SLPIELISA. CTE supernatants were analyzed for their $\beta$-defensins 1 and 2 content using an ELISA Development Kit (PeproTech, London, UK) according to the manufacturer's instructions. Briefly, plates were coated with anti- $\beta$-defensins 1 and 2 capture Ab. After washing the samples, the standards were added to the plate and the signal was detected with a detection $\mathrm{Ab}$ and Avidinhorseradish peroxidase conjugate. Plates were analyzed using an ELISA microplate reader Bio-Rad 680 (Bio-Rad Laboratories).

CTE supernatants were also analyzed for SLPI content using the Quantikine immunoassay Kit (R\&D Systems) according to the manufacturer's instructions. Briefly, supernatants were diluted 1:400 in diluent buffer. Plates were analyzed using an ELISA microplate reader Bio-Rad 680 (Bio-Rad Laboratories).

Statistical analysis. Each data point related to a single CTE represents the pooled value of 16 tissue blocks cultured under the same conditions. The statistical significance of differences between results from different experimental groups was evaluated with the paired or unpaired Student's $t$-test where appropriate. The pooled data are presented as means \pm s.e.m.. All of the hypothesis tests were twotailed, and a $P$ value of $\leqslant 0.05$ defined statistical significance.

Ethical declaration. This study was conducted in observation with the Declaration of Helsinki and after signing an informed consent form before the initiation of the study.

\section{ACKNOWLEDGEMENTS}

E.S. conducted this study as student of the Program of Basic and Applied Immunology, International PhD School in Molecular Medicine, San Raffaele University, Milan, Italy; E.S.'s PhD fellowship was supported by a grant of the Cariplo Foundation, Milano, Italy. This study was supported by the grant AIDS $40 \mathrm{H} 77$ of the National Program of Research on HIV/AIDS of the Italian Ministry of Health (to G.P.). The work of A.L., J-C.G., and L.M. was supported by the NICHD Intramural Program. We thank Elisa Vicenzi for helpful discussions.

\section{DISCLOSURE}

The authors declared no conflict of interest.

c 2013 Society for Mucosal Immunology

\section{REFERENCES}

1. Lisco, A. et al. Semen of HIV-1-infected individuals: local shedding of herpesviruses and reprogrammed cytokine network. J. Infect. Dis. 205, 97-105 (2012).

2. Grivel, J.C., Shattock, R.J. \& Margolis, L.B. Selective transmission of R5 HIV-1 variants: where is the gatekeeper?. J. Transl. Med. 9 (Suppl 1), S6 (2011).

3. Abdool Karim, Q. et al. Effectiveness and safety of tenofovir gel, an antiretroviral microbicide, for the prevention of HIV infection in women. Science 329, 1168-1174 (2010).

4. Quinones-Mateu, M.E. \& Vanham, G. HIV microbicides: where are we now?. Curr. HIV Res. 10, 1-2 (2012).

5. Sugaya, M., Lore, K., Koup, R.A., Douek, D.C. \& Blauvelt, A. HIV-infected Langerhans cells preferentially transmit virus to proliferating autologous CD4 + memory T cells located within Langerhans cell-T cell clusters. J. Immunol. 172, 2219-2224 (2004).

6. Shen, R. et al. Macrophages in vaginal but not intestinal mucosa are monocyte-like and permissive to human immunodeficiency virus type 1 infection. J. Virol. 83, 3258-3267 (2009).

7. Duncan, C.J. \& Sattentau, Q.J. Viral determinants of HIV-1 macrophage tropism. Viruses 3, 2255-2279 (2011).

8. Wira, C.R., Fahey, J.V., Sentman, C.L., Pioli, P.A. \& Shen, L. Innate and adaptive immunity in female genital tract: cellular responses and interactions. Immunol. Rev. 206, 306-335 (2005).

9. Sun, L. et al. Human beta-defensins suppress human immunodeficiency virus infection: potential role in mucosal protection. J. Virol. 79, 14318-14329 (2005).

10. Wira, C.R., Patel, M.V., Ghosh, M., Mukura, L. \& Fahey, J.V. Innate immunity in the human female reproductive tract: endocrine regulation of endogenous antimicrobial protection against HIV and other sexually transmitted infections. Am. J. Reprod. Immunol. 65, 196-211 (2011).

11. Keller, M.J. et al. PRO 2000 elicits a decline in genital tract immune mediators without compromising intrinsic antimicrobial activity. AIDS 21, 467-476 (2007).

12. Wira, C.R. \& Fahey, J.V. A new strategy to understand how HIV infects women: identification of a window of vulnerability during the menstrual cycle. AIDS 22, 1909-1917 (2008). 
13. Wira, C.R. et al. Sex hormone regulation of innate immunity in the female reproductive tract: the role of epithelial cells in balancing reproductive potential with protection against sexually transmitted pathogens. Am. J. Reprod. Immunol. 63, 544-565 (2010).

14. Hel, Z., Stringer, E. \& Mestecky, J. Sex steroid hormones, hormonal contraception, and the immunobiology of human immunodeficiency virus1 infection. Endocr. Rev. 31, 79-97 (2010).

15. Wicherek, L. etal. The characterization of the subpopulation of suppressive $\mathrm{B} 7 \mathrm{H} 4(+)$ macrophages and the subpopulation of $\mathrm{CD} 25(+) \mathrm{CD} 4(+)$ and FOXP3 $(+)$ regulatory T-cells in decidua during the secretory cycle phase, Arias Stella reaction, and spontaneous abortion — a preliminary report. Am. J. Reprod. Immunol. 61, 303-312 (2009).

16. Chao, K.H., Wu, M.Y., Chen, M.J., Hung, C.C. \& Ho, H.N. Impact of the menstrual cycle on immunologic markers in HIV-infected Taiwanese women. Taiwan J. Obstet. Gynecol. 45, 42-47 (2006).

17. Vishwanathan, S.A. et al. High susceptibility to repeated, low-dose, vaginal SHIV exposure late in the luteal phase of the menstrual cycle of pigtail macaques. J. Acquir. Immune Defic. Syndr. 57, 261-264 (2011).

18. Poonia, B. et al. Cyclic changes in the vaginal epithelium of normal rhesus macaques. J. Endocrinol. 190, 829-835 (2006).

19. Heffron, R. et al. Use of hormonal contraceptives and risk of HIV-1 transmission: a prospective cohort study. Lancet Infect. Dis. 12, 19-26 (2012).

20. Tebit, D.M., Ndembi, N., Weinberg, A. \& Quinones-Mateu, M.E. Mucosal transmission of human immunodeficiency virus. Curr. HIV Res. 10, 3-8 (2012)

21. Greenhead, P. et al. Parameters of human immunodeficiency virus infection of human cervical tissue and inhibition by vaginal virucides. J. Virol. 74, 5577-5586 (2000).

22. Saba, E. et al. HIV-1 sexual transmission: early events of HIV-1 infection of human cervico-vaginal tissue in an optimized ex vivo model. Mucosal Immunol. 3, 280-290 (2010).

23. Thompson, K.A., Cherry, C.L., Bell, J.E. \& McLean, C.A. Brain cell reservoirs of latent virus in presymptomatic HIV-infected individuals. Am. J. Pathol. 179, 1623-1629 (2011).

24. Alexaki, A., Liu, Y. \& Wigdahl, B. Cellular reservoirs of HIV-1 and their role in viral persistence. Curr. HIV Res. 6, 388-400 (2008).

25. Vicenzi, E. et al. Divergent regulation of HIV-1 replication in PBMC of infected individuals by CC chemokines: suppression by RANTES, MIP1 alpha, and MCP-3, and enhancement by MCP-1. J. Leukoc. Biol. 68, 405-412 (2000).

26. Fantuzzi, L. et al. Endogenous CCL2 (monocyte chemotactic protein-1) modulates human immunodeficiency virus type-1 replication and affects cytoskeleton organization in human monocyte-derived macrophages. Blood 102, 2334-2337 (2003).

27. Shen, R., Richter, H.E. \& Smith, P.D. Early HIV-1 target cells in human vaginal and ectocervical mucosa. Am. J. Reprod. Immunol. 65, 261-267 (2011).

28. Pohlmann, S., Baribaud, F. \& Doms, R.W. DC-SIGN and DC-SIGNR: helping hands for HIV. Trends Immunol. 22, 643-646 (2001).

29. Geijtenbeek, T.B. \& van Kooyk, Y. DC-SIGN: a novel HIV receptor on DCs that mediates HIV-1 transmission. Curr. Top. Microbiol. Immunol. 276, 31-54 (2003).

30. Gringhuis, S.I. et al. HIV-1 exploits innate signaling by TLR8 and DC-SIGN for productive infection of dendritic cells. Nat. Immunol. 11, 419-426 (2010).
31. Berzi, A. et al. A glycomimetic compound inhibits DC-SIGN-mediated HIV infection in cellular and cervical explant models. AIDS 26, 127-137 (2012).

32. Lehner, T. The role of CCR5 chemokine ligands and antibodies to CCR5 coreceptors in preventing HIV infection. Trends Immunol. 23, 347-351 (2002).

33. Iqbal, S.M. et al. Elevated T cell counts and RANTES expression in the genital mucosa of HIV-1-resistant Kenyan commercial sex workers. J. Infect. Dis. 192, 728-738 (2005).

34. Swingler, S. et al. HIV-1 Nef mediates lymphocyte chemotaxis and activation by infected macrophages. Nat. Med. 5, 997-1003 (1999).

35. Dai, L. \& Stevenson, M. A novel motif in HIV-1 Nef that regulates MIP-1 beta chemokine release in macrophages. J. Virol. 84, 8327-8331 (2010).

36. D'Aversa, T.G., Yu, K.O. \& Berman, J.W. Expression of chemokines by human fetal microglia after treatment with the human immunodeficiency virus type 1 protein Tat. J. Neurovirol. 10, 86-97 (2004).

37. Choe, W., Volsky, D.J. \& Potash, M.J. Induction of rapid and extensive beta-chemokine synthesis in macrophages by human immunodeficiency virus type 1 and gp120, independently of their coreceptor phenotype. J. Virol. 75, 10738-10745 (2001).

38. Sheffield, J.S., Wendel, G.D. Jr., McIntire, D.D. \& Norgard, M.V. The effect of progesterone levels and pregnancy on HIV-1 coreceptor expression. Reprod. Sci. 16, 20-31 (2009).

39. Meditz, A.L. et al. CCR5 expression is elevated on endocervical CD4 + T cells in healthy postmenopausal women. J. Acquir. Immune Defic. Syndr. 59, 221-228 (2012).

40. Salle, B. et al. Infection of macaques after vaginal exposure to cell-associated simian immunodeficiency virus. J. Infect. Dis. 202, 337-344 (2010).

41. Ma, Z., Lu, F.X., Torten, M. \& Miller, C.J. The number and distribution of immune cells in the cervicovaginal mucosa remain constant throughout the menstrual cycle of rhesus macaques. Clin. Immunol. 100, 240-249 (2001).

42. Ghosh, M. et al. Anti-HIV activity in cervical-vaginal secretions from HIVpositive and -negative women correlate with innate antimicrobial levels and IgG antibodies. PloS One 5, e11366 (2010).

43. Han, J.H. et al. Modulation of human beta-defensin-2 expression by 17beta-estradiol and progesterone in vaginal epithelial cells. Cytokine 49, 209-214 (2010).

44. Weinberg, A., Quinones-Mateu, M.E. \& Lederman, M.M. Role of human beta-defensins in HIV infection. Adv. Dent. Res. 19, 42-48 (2006).

45. Grivel, J.C. \& Margolis, L. Use of human tissue explants to study human infectious agents. Nat. Protoc. 4, 256-269 (2009).

46. Koup, R.A., Ho, D.D., Poli, G. \& Fauci, A.S. Isolation and quantitation of HIV in peripheral blood. Curr. Protoc. Immunol. Chapter 12, 12.2, 1-11 (2001).

47. Glushakova, S., Baibakov, B., Margolis, L.B. \& Zimmerberg, J. Infection of human tonsil histocultures: a model for HIV pathogenesis. Nat. Med. 1, 1320-1322 (1995).

48. Vicenzi, E., Panina-Bodignon, P., Vallanti, G., Di Lucia, P. \& Poli, G. Restricted replication of primary HIV-1 isolates using both CCR5 and CXCR4 in Th2 but not in Th1 CD4(+) T cells. J. Leukoc. Biol. 72, 913-920 (2002).

49. Collin, M. \& Gordon, S. The kinetics of human immunodeficiency virus reverse transcription are slower in primary human macrophages than in a lymphoid cell line. Virology 200, 114-120 (1994).

50. Biancotto, A. et al. A highly sensitive and dynamic immunofluorescent cytometric bead assay for the detection of HIV-1 p24. J. Virol. Methods 157, 98-101 (2009). 